\title{
OPEN Novel approach to modeling high-frequency activity data to assess therapeutic effects of analgesics in chronic pain conditions
}

\author{
Zekun Xu ${ }^{1}$, Eric Laber ${ }^{1}$, Ana-Maria Staicu ${ }^{1} \&$ B. Duncan X. Lascelles ${ }^{2,3,4,5}$
}

Osteoarthritis $(O A)$ is a chronic condition often associated with pain, affecting approximately fourteen percent of the population, and increasing in prevalence. A globally aging population have made treating $\mathrm{OA}$-associated pain as well as maintaining mobility and activity a public health priority. $\mathrm{OA}$ affects all mammals, and the use of spontaneous animal models is one promising approach for improving translational pain research and the development of effective treatment strategies. Accelerometers are a common tool for collecting high-frequency activity data on animals to study the effects of treatment on pain related activity patterns. There has recently been increasing interest in their use to understand treatment effects in human pain conditions. However, activity patterns vary widely across subjects; furthermore, the effects of treatment may manifest in higher or lower activity counts or in subtler ways like changes in the frequency of certain types of activities. We use a zero inflated Poisson hidden semi-Markov model to characterize activity patterns and subsequently derive estimators of the treatment effect in terms of changes in activity levels or frequency of activity type. We demonstrate the application of our model, and its advance over traditional analysis methods, using data from a naturally occurring feline $O A$-associated pain model.

To anyone who suffers chronic and persistent musculoskeletal pain, the negative impact on their quality of life is constant. In the U.S., more than 100 million people (nearly one third of the population) suffer from persistent pain with an associated economic cost of $\$ 600$ billion USD annually; this cost exceeds that of cardiovascular disease, cancer, and diabetes combined ${ }^{1}$. The most significant contribution to this cost comes from the impact of osteoarthritis (OA) and other musculoskeletal pain ${ }^{1}$. OA results in the deterioration of all components of joints ${ }^{2}$ and is often associated with pain. OA-associated pain results in significant morbidity and economic costs ${ }^{3}$; these costs, an aging population, and the growing knowledge of the health and psychological benefits of maintaining mobility and activity, have made the treatment of OA and the associated pain a public health priority ${ }^{4-8}$. However, recent review papers show that the current practice of translational biomedical research is not producing new therapeutics for pain control in humans ${ }^{9-11}$. While these reviews highlight the lack of translation of basic research into new approved therapeutics for treatment of persistent pain in humans, they also discuss how the processes involved could be optimized to improve the chances of successful translation, including discussion of improved models and more relevant outcome measures. As OA and associated pain affects all mammals, the study of OA in non-human animals is both important in its own right (to increase the function and quality of life in affected animals) and for its ability to generate new knowledge about the treatment of OA in humans. Recently, the use of naturally occurring OA in pet animals has been suggested as a means of helping to improve translational research for the development of analgesic therapeutics in humans ${ }^{12}$. A significant advantage of naturally occurring disease in pets as a model of human conditions is the variation and complexity of the model. Measurement of spontaneous activity as an outcome measure may be particularly relevant to translational work as spontaneous activity

${ }^{1}$ Department of Statistics, North Carolina State University, Raleigh, NC, USA. ${ }^{2}$ Comparative Pain Research and Education Center, College of Veterinary Medicine, North Carolina State University, Raleigh, NC, USA. ${ }^{3}$ Translational Research in Pain (TRiP) Program, North Carolina State University, College of Veterinary Medicine, Raleigh, NC, USA. ${ }^{4}$ Thurston Arthritis Center, UNC School of Medicine, Chapel Hill, NC, USA. ${ }^{5}$ Center for Translational Pain Research, Department of Anesthesiology, Duke University, Durham, NC, USA. ${ }^{\bowtie}$ email: dxlascel@ncsu.edu 
may relate to spontaneous pain-something that has been difficult to model in animals. Just as the variability in naturally occurring disease is an advantage, the variability in clinically meaningful outcome measures such as activity is a challenge. This could be the reason for the lack of use of activity as a functional outcome measure in pain studies in humans, despite the importance of mobility and activity to quality of life ${ }^{7}$. The work we are reporting is motivated by our involvement in studies of OA-associated pain and the treatment effects on mobility and activity in domestic cats ${ }^{13,14}$. We posit that the methodology we describe presents a significant advance in how such activity data are analyzed when used as an outcome measure and has relevance to both large animal models and clinical evaluation in humans.

The current study uses data from a randomized cross-over study designed to evaluate the effectiveness of meloxicam, a nonsteroidal anti-inflammatory drug (NSAID), in owned, pet adult domestic cats with OA-associated pain $^{15}$. In this study, cat activity patterns were measured at one-minute intervals using an omnidirectional accelerometer ${ }^{16}$. Accelerometer readings are integers quantifying the intensity of change in acceleration over the preceding, pre-defined epoch. Thus, for each subject, the accelerometer produces high-frequency, integer-valued, longitudinal data. The main goal of the present study is to re-evaluate the use of and analysis of activity data to determine whether meloxicam is effective in reducing OA-associated pain. We aimed to use the objectively measured physical activity recorded via accelerometers and analyze these data using a novel zero-inflated hidden Poisson semi-Markov model. The primary hypothesis of the present study is that meloxicam reduces OAassociated pain and this manifests through increased activity during more intense activity during the treatment period relative to the placebo period. One common approach for the analyses of such data is to aggregate the observed accelerometer data within each subject and treatment condition and subsequently to use an ANOVA to compare treatment with control ${ }^{15-17}$. Alternatively, one could aggregate the data over a shorter time interval, e.g., a day, and model the aggregated process using methods for smooth longitudinal data ${ }^{18}$. However, such aggregation can obscure changes in behavior patterns which do not produce a large difference in mean activity. For example, an effective treatment that reduces pain may lead to higher quality rest (more zero activity readings), but also more high-intensity movement (more high activity readings), potentially producing no change in mean activity. High volume activity data has the potential to be a more sensitive outcome measure, but thus far, analysis of such complex, high-volume multidirectional effects on activity, that takes into account the wide variation in individual activity patterns, has not been developed.

We propose modeling the minute-by-minute accelerometer data using a hidden semi-Markov model. Such a model is scientifically appealing in that the hidden states can be viewed as corresponding to latent (unobserved) activities, e.g., running, walking, resting, etc., and state duration corresponds to the length of time a subject is engaged in a given activity. Latent state-space models are common in the analysis of mobility data measured using wearable computing ${ }^{19-23}$. In the context of treatment evaluation, hidden Markov models have also been used to model latent health states and subsequently conduct inference for activity patterns in terms of transitions among these states and activity patterns within each state ${ }^{24,25}$; however, previous applications have considered relatively coarse time scales, e.g., daily or weekly measurements, and subsequently low data volume. In the current application, subject activity is measured every minute and as a result each subject has more than one-hundred thousand measurements during the observation period. Such high volume allows for flexible modeling and estimation of distinct parameters for each subject; this is important as activity patterns can vary widely across subjects.

In the current example of the spontaneous cat OA-pain model, more than $70 \%$ of the observations are zero and therefore we propose a zero-inflated hidden Poisson semi-Markov model with patient-specific intensities in each latent state. The proposed model is an extension of a zero-inflated Poisson hidden Markov model ${ }^{26,27}$ to the hidden semi-Markov model framework that allows for distinct state duration densities ${ }^{28-30}$. Furthermore, to facilitate computationally efficient estimation with high-frequency data, we propose a two stage estimation procedure $^{31,32}$ that can be used with millions of observations without specialized computing resources.

The data for this work come from a study of the treatment effects of meloxicam (a non-steroidal anti-inflammatory drug) in a feline model of spontaneous OA-associated pain ${ }^{15,33}$. This study was a randomized, doubleblind, placebo-controlled, crossover study to evaluate the effectiveness of meloxicam treatment to improve mobility and function in owned, pet cats with OA-associated pain. This study was approved by the Animal Care and Use Committee (Protocol \# 11-102-O) at North Carolina State University College of Veterinary Medicine (NCSU-CVM), and written owner consent was granted in each case following verbal discussion of the study.

\section{Results}

Figure 1 displays the daily activity profile for a typical subject. There are multiple prolonged intervals in which there is no movement. In total, more than $70 \%$ of the minute-by-minute activity counts are zero; furthermore, the non-zero counts are heavily right-skewed owing to infrequent but high-intensity activities. Thus, such cat accelerometer data are heavy-tailed with an excessive proportion of zeros.

Figure 2 displays mean activity counts for each subject across each period of the study. In this figure, each gray line shows the mean activity count for a single subject in the study, and the black line shows the overall mean activity count for all subjects in their associated treatment group. The left panel shows data for the subjects that were randomized to meloxicam in the first treatment period, whereas the right panel shows data for the subjects that were randomized to placebo in the first treatment period. In both panels, one can see that the overall mean activity count is higher under meloxicam than placebo, but only marginally. A total of 41 out of 58 cats have a numerically larger mean activity count in the meloxicam period than in the placebo period. However, there is large between-subject variability. The standard errors for the group mean activity count at each treatment period range from 16.7 to 18.1 .

We applied the proposed zero-inflated hidden semi-Markov model (detailed in the Methods section) to the longitudinal accelerometer data from this study of the treatment effect of meloxicam. We chose the number of 

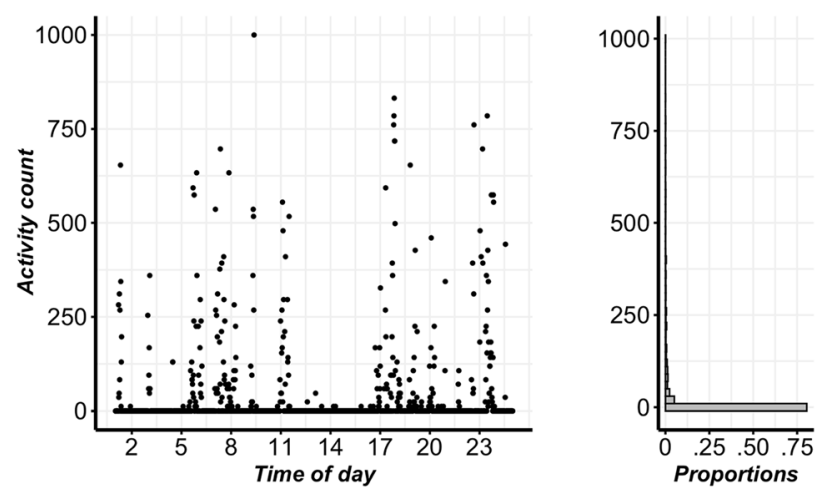

Figure 1. Left: Sample daily accelerometer data for a typical cat in the study of the treatment effect of meloxicam, showing the crepuscular pattern of activity expected in cats. Right: Histogram showing the proportions of time spent in different levels of activity during a $24 \mathrm{~h}$ period.
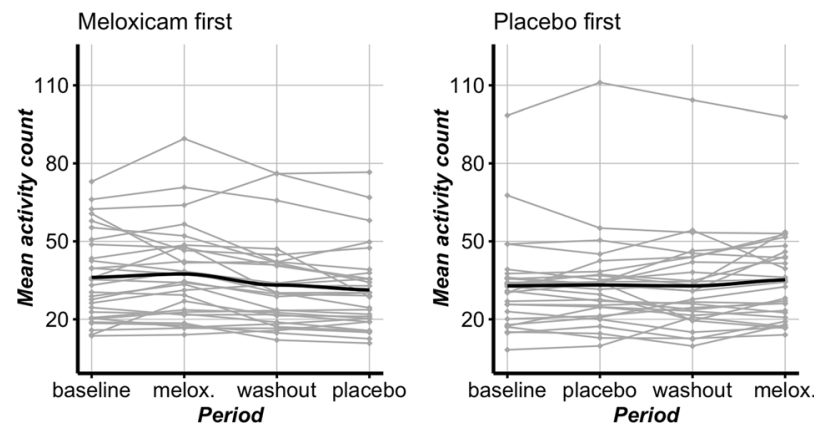

Figure 2. Plots of the mean activity count over each period for each subject separated by treatment group. The dark line in each panel is the group mean activity count.

\begin{tabular}{|l|l|l|l|}
\hline Parameter & Estimate & Standard error & $\boldsymbol{p}$ value \\
\hline State 1: odds of zero & -0.0299 & 0.0433 & 0.4899 \\
\hline State 1: mean activity count & 0.0222 & 0.0181 & 0.2187 \\
\hline State 2: mean activity count & 0.0444 & 0.0311 & 0.1538 \\
\hline State 3: mean activity count & 0.0524 & 0.0318 & 0.0990 \\
\hline State 4: mean activity count & 0.0914 & 0.0351 & 0.0091 \\
\hline State 5: mean activity count & 0.0819 & 0.0235 & 0.0005 \\
\hline State 6: mean activity count & 0.0728 & 0.0260 & 0.0051 \\
\hline
\end{tabular}

Table 1. Summary of treatment effects (meloxicam vs. placebo) in each latent activity state in the increasing order of mean activity count. Actual activities represented in each state are unknown, but for instance, state 1 may include resting and other activities with the lowest mean activity count, while state 6 may include jumping and other activities with the highest mean activity count. At significance level 0.05 , there is significant positive treatment effect in state $4,5,6$, i.e. higher level activity intensity states.

latent activity states to be six using minimum Bayesian information criterion (BIC) as our data-driven selection method. The covariates included in the model were: treatment status, weekend (yes/no), night (yes/no), sex, Body Condition Score (BCS), age, total OA score, and treatment sequence.

Table 1 displays the estimated treatment effects in terms of mean activity counts within each latent state. There are significant effects $(p<0.05)$ in latent states $4-6$ suggesting that cats treated with meloxicam may have increased mean activity while engaged in more intensive activities (e.g., running, jumping, etc.). The effect size of the treatment on those more intensive activities ranges from 7.28 to $9.14 \%$. Table 2 shows the mean proportion of time spent in each latent state estimated using the Viterbi algorithm ${ }^{34}$. It can be seen that the proportion of time spent in each activity is similar across the treatment and placebo periods. The proportion of time spent is each latent activity states is similar before and after the meloxicam treatment (Table 2); what significantly changes is the mean activity counts in more intense states (4-6), i.e., cats become more active in those intense activity 


\begin{tabular}{|l|l|l|}
\hline Activity state & Meloxicam (\%) & Placebo (\%) \\
\hline State 1 & 83.6 & 84.5 \\
\hline State 2 & 5.5 & 5.3 \\
\hline State 3 & 4.5 & 4.3 \\
\hline State 4 & 3.4 & 3.3 \\
\hline State 5 & 2.1 & 1.9 \\
\hline State 6 & 0.9 & 0.8 \\
\hline
\end{tabular}

Table 2. Mean proportion of time spent in each decoded activity state. The latent states are in increasing order of mean activity count. Actual activities represented in each state are unknown, but for instance, state 1 may include resting and other activities with the lowest mean activity count, while state 6 may include jumping and other activities with the highest mean activity count. At significance level 0.05 , there is significant positive treatment effect in state $4,5,6$, i.e. higher level activity intensity states.

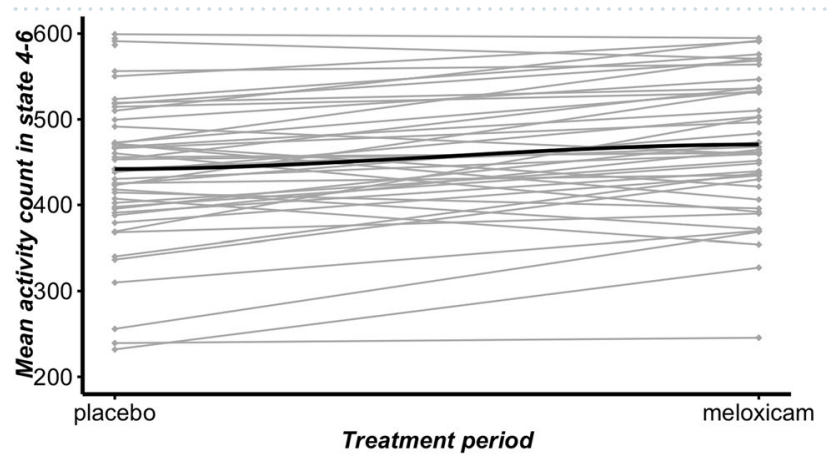

Figure 3. Mean activity count for each subject in the placebo and meloxicam treatment period for latent state 4-6. The dark line in each panel is the overall mean activity count. The mean increase in the activity count is 23.0, with a standard error of 7.2.

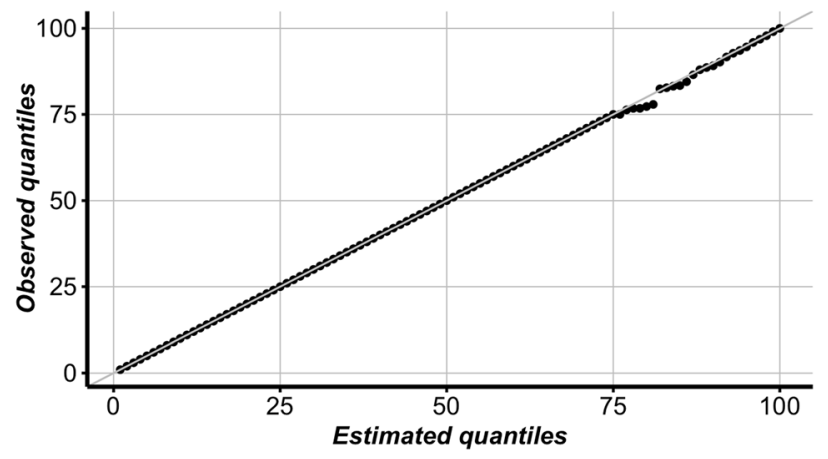

Figure 4. Estimated quantiles based on fitted model versus observed quantiles. In both distributions, from the 1st up to the 75th quantiles are zero. The rest of the quantiles also match well with each other, which indicates there is no lack of fit in the proposed model.

states (Table 1). The mean activity count for each subject in the placebo and meloxicam treatment period for latent state 4-6 is shown in Fig. 3.

Figure 4 shows a QQ-plot of the estimated quantiles using the fitted model and the observed quantiles of the activity counts. It can be seen that they follow each other closely and that there are no indications of serious lack-of-fit.

\section{Discussion}

We proposed a zero-inflated Poisson hidden semi-Markov model for activity data captured by accelerometer readings in a naturally occurring model of OA-associated pain. The proposed model can be used to characterize the impact of treatment on activity including changes in activity duration, intensity, and frequency. In the context of OA-associated pain, understanding such impacts is critical to fully understanding the effects of treatment on patient mobility and therefore quality of life. 
Our method presents a significant advance in the analysis of therapeutic efficacy using activity counts as compared to the original analysis in Gruen et $\mathrm{al}^{15}$ as well as subsequent work in Gruen et $\mathrm{al}^{18}$. In Gruen et $\mathrm{al}^{15}$, the activity counts were averaged within each treatment period for each cat, and an ANOVA model was used to determine the effectiveness of meloxicam. Their analysis showed a non-significant increase $(p=0.31)$ in the activity counts among the cats that received meloxicam in the first treatment period. This may be due to the effect of data aggregation method, which can smooth away important effects and reduce statistical power. Gruen et al ${ }^{18}$ aggregated the counts across days and then used functional data analysis methods to formally assess treatment effect. This method failed to identify a significant difference between normal and arthritic cats $(p=0.541)$ due to the same limitations discussed above.

In contrast, our proposed subject-specific method not only preserves the original data 'as is' but also accounts for the large between-subject variability. More importantly, our approach can analyze the conditional treatment effect in each activity state. Indeed, our model found that meloxicam significantly improves the mobility of cats in more intensive activities, while the activity patterns did not change in less intensive activities. Although an easy assumption is that the increase in more intense activities is beneficial, the clinical relevance of these findings is, at present, unknown.

Our approach is relevant to humans. Cats, like people, show patterns of activity, with large periods of relative or absolute inactivity. Cats are generally crepuscular-that is, they show phasic patterns of activity, as do humans. Such zero-inflated patterned activity is thus common to cats and humans, and so our approach is relevant to human activity data. As the approach does not need to know what activity is being performed, it negates the need for algorithms to detect specific activities.

Future work could support our approach by evaluating concurrent video capture of moving subjects to verify that as more intense activities are being performed, the relevant latent states change. Additionally, research is needed to define the clinical relevance of the changes we have detected using the zero-inflated hidden Poisson semi-Markov model.

Our refinement of the analysis of high-volume activity data in this naturally occurring model of pain provides a clinically relevant outcome measure that can be used in naturally occurring models osteoarthritis in companion animals to provide highly relevant data on the potential efficacy of putative drugs prior to Phase II and III efficacy studies in humans, thus improving the translational paradigm by optimizing the critical go/no-go decision prior to Phase II. Such sophisticated approaches to high-volume activity data have not been applied to humans and our approach also has relevance to the analysis of activity data in humans to better understand the impact of therapeutics in a more refined manner.

\section{Methods}

The original study was approved by the Animal Care and Use Committee (Protocol \# 11-102-O) at North Carolina State University College of Veterinary Medicine (NCSU-CVM), and written owner consent was granted in each case following verbal discussion of the study. The study was performed in accordance with the relevant guidelines and regulations. Full details of the study have been previously published ${ }^{15}$ and pertinent methodological information is provided in Supplementary File 1.

A total of 66 subjects were enrolled in the original study of which 58 had available accelerometer measurements from both arms of the cross-over design. Subjects were given placebo during the open label baseline period (Weeks 1 and 2). In the first blinded treatment period (Weeks 3, 4, and 5) half of the subjects were randomized to receive meloxicam and the remainder were randomized to receive placebo. At the end of the first blinded phase, subjects entered a three-week blinded washout (Weeks 6, 7, and 8) before switching treatments for the second blinded phase. All subjects wore an accelerometer (Actical) on their collar throughout the entire study, with the epoch set at $1 \mathrm{~min}$, providing an activity count for every minute of the 11-week study for each subject.

Zero-inflated Poisson hidden semi-Markov model. We assume that the observed data are of the form $\left\{\left(\mathrm{W}_{\mathrm{i}}, \mathrm{Z}_{\mathrm{i}}^{1}, \mathrm{X}_{\mathrm{i}}^{1}, \mathrm{Y}_{\mathrm{i}}^{1}, \ldots, \mathrm{Z}_{\mathrm{i}}^{\mathrm{T}}, \mathrm{X}_{\mathrm{i}}^{\mathrm{T}}, \mathrm{Y}_{\mathrm{i}}^{\mathrm{T}}\right)\right\}$ and comprise $\mathrm{n}$ independent replicates of the trajectory $\left(\mathrm{W}, \mathrm{Z}^{1}, \mathrm{X}^{1}, \mathrm{Y}^{1}, \ldots, \mathrm{Z}^{\mathrm{T}}\right.$, $\left.\mathrm{X}^{\mathrm{T}}, \mathrm{Y}^{\mathrm{T}}\right)$, where: $W \in \mathbb{R}^{d}$ denotes the baseline (pre-treatment) subject characteristics; $\mathrm{T}$ is a fixed time horizon; $Z^{t} \in \mathbb{R}^{p}$ denotes environmental factors at $\mathrm{t}=1, \ldots, \mathrm{T} ; X^{t} \in \mathbb{R}^{q}$ encodes treatment administered at time $\mathrm{t}=1$, $\ldots, \mathrm{T}$; and $Y^{t} \in \mathbb{N}$ denotes the integer accelerometer activity reading at time $t=1, \ldots, \mathrm{T}$. In our application, $Z^{t} \in\{0,1\}^{2}$ comprises indicators coding period of day and weekend versus weekday, and $X^{t} \in\{0,1\}^{1}$ encodes treatment indicators (active versus placebo). While we develop our models allowing for rather general environmental factors and treatment processes, having binary factors in our application makes it simpler to enforce a stochastic ordering on the distributions indexed by latent states which is important for interpretability and coherence of the fitted models, e.g., if one wants the latent states ordered by mean activity intensity within each subject and to have these latent states align across subjects. We model the evolution of the accelerometer data using a zero-inflated Poisson hidden semi-Markov model which develop in the remainder of this subsection.

Let $S_{i}^{t} \in\{1, \ldots, M\}$ denote the latent state, i.e., the unobserved activity type, of subject $\mathrm{i}=1, \ldots, \mathrm{n}$ at time $\mathrm{t}=1, \ldots, \mathrm{T}$. At every single minute, each subject is assumed to be in one of the $\mathrm{M}$ latent activity states, with the convention that " 1 " represents a state where a low level of physical activity is typical; " 2 " represents a state where higher level activity is typical and so forth. The duration in each state is characterized by state-specific parameters and estimated from the data.

We assume the latent states evolve according to a semi-Markov model indexed by: (i) an initial state distribution, $\delta_{m, i}=P\left(S_{i}^{1}=m\right)$ for $\mathrm{m}=1, \ldots, \mathrm{M}$; (ii) state duration distributions $r_{m, i}\left(v ; x^{t}, z^{t}\right)=P\left(S_{i}^{t+1}=m, \ldots, S_{i}^{t+v} \neq m \mid S_{i}^{t}=m, X_{i}^{t}=x^{t}, Z_{i}^{t}=z^{t}\right)$ for $\mathrm{v}>0, \mathrm{~m}=1, \ldots, \mathrm{M}$; and (iii) the transition probabilities $Q_{i}\left(m, l ; x^{t}, z^{t}\right)=P\left(S_{i}^{t+1}=l \mid S_{i}^{t}=m, S_{i}^{t+1} \neq m, X_{i}^{t}=x^{t}, Z_{i}^{t}=z^{t}\right)$ for $m, l=1, \ldots, M$. 
We model the initial state distribution nonparametrically using sample frequencies of the estimated latent states. We assume a multinomial logistic regression model for the transition probabilities so that

$$
Q_{i}\left(m, l ; x^{t}, z^{t}\right)=\frac{\exp \left(d_{m, l, i}^{\prime} x^{t}+\varrho_{m, l, i}^{\prime} z^{t}\right)}{\sum_{k=1}^{M} \exp \left(d_{m, k, i}^{\prime} x^{t}+\varrho_{m, k, i}^{\prime} z^{t}\right)},
$$

where $d_{m, k, i}$ and $\varrho_{m, k, i}$ are unknown coefficient vectors for $\mathrm{m}, \mathrm{k}=1, \ldots, \mathrm{M}$. We assume that the state duration distribution follows a latent accelerated failure time model so that.

$$
r_{m, i}\left(v ; x^{t}, z^{t}\right)=\exp \left(c_{m, i}^{\prime} x^{t}+\eta_{m, i}^{\prime} z^{t}\right) \int_{v}^{v+1} f_{m, i}\left\{\exp \left(c_{m, i}^{\prime} x^{t}+\eta_{m, i}^{\prime} z^{t}\right) u\right\} d u
$$

where $f_{m, i}$ is a base-density corresponding to a null treatment condition (coded as $x^{t} \equiv 0$ ) and $c_{m, i}, \eta_{m, i}$ are unknown coefficient vectors for $m=1, \ldots, M$. We further assume that $Y_{i}^{t} \perp\left(Z_{i}^{1}, X_{i}^{1}, S_{i}^{1}, \ldots, Z_{i}^{t-1}, X_{i}^{t-1}, S_{i}^{t-1}\right) \mid\left(Z_{i}^{t}, X_{i}^{t}, S_{i}^{t}\right)$ and that the accelerometer activity readings are distributed as.

$$
P\left(Y_{i}^{t}=y \mid S_{i}^{t}=1, Z_{i}^{t}=z, X_{i}^{t}=x\right)=p_{i}^{t}(z, x) I_{y=0}+\left\{1-p_{i}^{t}(z, x)\right\} \frac{\left\{\lambda_{i}^{t}(m, z, x)\right\}^{y} \exp \left\{\left\{\lambda_{i}^{t}(m, z, x)\right\}\right.}{y !},
$$

when the subject is in the latent state 1 , and

$$
P\left(Y_{i}^{t}=y \mid S_{i}^{t}=m, Z_{i}^{t}=z, X_{i}^{t}=x\right)=\frac{\left\{\lambda_{i}^{t}(m, z, x)\right\}^{y} \exp \left\{\lambda_{i}^{t}(m, z, x)\right\}}{y !},
$$

when the subject is in latent state $\mathrm{m}=2,3, \ldots, \mathrm{M}$. Thus, the accelerometer readings are modeled as a zero-inflated Poisson with intensity function $\lambda_{i}^{t}: S \times \mathbb{R}^{q} \times \mathbb{R}^{p} \rightarrow \mathbb{R}_{+}$and weight functions $p_{i}^{t}: S \times \mathbb{R}^{q} \times \mathbb{R}^{p} \rightarrow[0,1]$ for $i=1, \ldots, n, t=1, \ldots, T$. Furthermore, for each subject $i$, time $t$, and latent state $m$, we assume that these functions are of the form

$$
\begin{aligned}
& \log \left\{\frac{p_{i}^{t}(z, x)}{1-p_{i}^{t}(z, x)}\right\}=b_{0,0, i}+b_{1,0, i}^{\prime} x+\gamma_{0, i}^{\prime} z, \\
& \log \left\{\lambda_{i}^{t}(m, z, x)\right\}=b_{0, m, i}+b_{1, m, i}^{\prime} x+\gamma_{m, i}^{\prime} z,
\end{aligned}
$$

where $b_{0, k, i}, b_{1, k, i}, \gamma_{k, i}, \mathrm{k}=0,1, \ldots, \mathrm{M}$ are unknown coefficient vectors. In our application, in which $x^{t}$ and $z^{t}$ are represented as binary vectors, we impose the constraints $b_{0, m+1, i}>b_{0, m, i}, b_{1, m+1, i} \geq b_{1, m, i}, \gamma_{m+1, i} \geq \gamma_{m, i}$ for $\mathrm{m}=1$, ..., $\mathrm{M}-1$. These constraints ensure that the intensity functions of the latent states are monotone increasing, i.e., $\lambda_{i}^{t}(m+1, z, x)>\lambda_{i}^{t}(m, z, x)$ for all $\mathrm{z}, \mathrm{x}$, and $\mathrm{m}$.

The preceding model describes individual level accelerometer data as a function of evolving covariate and treatment information. The idea of using the framework of hidden Markov model to analyze physical activity data can also be seen in the recent work of Huang et al. ${ }^{35}$ and van Kuppevelt et al. ${ }^{36}$. However, the cat accelerometer data we consider are much sparser than the human accelerometer data in their work, which motivates a zero-inflation component in our model. Moreover, we can include exogenous, time-varying variables like treatment period, night indicator, and weekend indicator in our model so as to explicitly address their effects on the activity patterns of cats. Our individual level model is a nontrivial extension of the zero-inflated Poisson hidden Markov model in the literature 26,27 , which not only allows for distinct state duration densities but also incorporates covariates and treatment in both transition probabilities and latent state durations.

To draw more general, i.e., population-level, inferences we model individual-level treatment effect parameters as functions of baseline subject information as follows. We assume that $\mathrm{E}\left(b_{1, m, i} \mid W_{i}=w\right)=\Omega_{m, 0}+\Omega_{m, 1} w$, where $\Omega_{m, 0} \in \mathbb{R}^{q}$ and $\Omega_{m, 1} \in \mathbb{R}^{q \times d}$ are matrices of unknown coefficients encoding treatment effect on the outcome for $\mathrm{m}=0,1, \ldots, \mathrm{M}$. Similarly, we assume that $\mathrm{E}\left(c_{m, i} \mid W_{i}=w\right)=\Gamma_{m, 0}+\Gamma_{m, 1} w$ and $\mathrm{E}\left(d_{m, l, i} \mid W_{i}=w\right)=\Lambda_{m, l, 0}+\Lambda_{m, l, 1} w$, where $\Gamma_{m, 0}, \Lambda_{m, l, 0} \in \mathbb{R}^{q}$ and where $\Gamma_{m, 1}, \Lambda_{m, l, 1} \in \mathbb{R}^{q \times d}$ encode the effect of treatment on state duration and transition probabilities for $m, l=1, \ldots, M$. The idea of aggregating individuallevel models to conduct population-level inference has been applied in other contexts including longitudinal and growth curve analyses ${ }^{31,37,38}$.

Estimation. We estimate the subject-specific parameters using maximum-likelihood estimation via the forward-backward recursive representation of the likelihood ${ }^{39}$; the number of latent states, $\mathrm{M}$, is selected using $\mathrm{BIC}^{38}$. Subsequently, the population-level parameters are estimated by regressing the subject-specific maximum likelihood estimators on baseline covariates using least squares. This two-stage approach, which will be detailed shortly, is computationally efficient for high-frequency data like those collected in the meloxicam study. Let

$$
\theta_{i}=\left\{\delta_{m}, d_{m, k, i}, \varrho_{m, k, i}, c_{m, i}, \eta_{m, i}, b_{0,0, i}, b_{0, m, i}, b_{1,0, i}, b_{1, m, i}, \gamma_{0, i}, \gamma_{m, i}\right\}_{m, k=1, \ldots, M}
$$

be the collection of subject-specific parameters for $\mathrm{i}=1, \ldots, \mathrm{n}$. We construct estimators $\hat{\theta}_{i, T}$ of $\theta_{i}$ by maximizing the log-likelihood (see supplementary file 2 for details). 
For $\mathrm{m}=0,1, \ldots, \mathrm{M}$, let $\hat{b}_{1, \mathrm{~m}, \mathrm{i}, \mathrm{T}}$ denote the maximum likelihood estimator of $b_{1, m, i}$. Subsequently, define $\hat{\Omega}_{m, 0, n}$, $\hat{\Omega}_{m, 1, n}=\operatorname{argmin}_{\Omega_{m, 0}, \Omega_{m, 1}} \sum_{i=1}^{n}\left\|\hat{b}_{1, m, i, T}-\Omega_{m, 0}-\Omega_{m, 1} W_{i}\right\|^{2}$ to be the two-stage estimator of $\Omega_{m, 0}, \Omega_{m, 1}$. The estimators $\hat{\Gamma}_{m, 0, n}, \hat{\Gamma}_{m, 1, n}, \hat{\Lambda}_{m, l, 0, n}, \hat{\Lambda}_{m, l, 1, n}$ of $\Gamma_{m, 0}, \Gamma_{m, 1}, \Lambda_{m, l, 0}, \Lambda_{m, l, 1}$ are defined analogously.

The statistical analysis in the manuscript is implemented using the software R version 2.0.6. We developed an R package "ziphsmm" to analyze such high-volume, zero-inflated accelerometer data, which is publicly available online (https://cran.r-project.org/web/packages/ziphsmm/index.html).

Theoretical properties. Throughout we use a star superscript, e.g., $\theta_{i}^{*}$ to denote the population analogue of the maximum likelihood estimator of a parameter indexing the proposed model; if the model is correctly specified, then under the conditions stated below, these parameters will correspond to the true parameters indexing the generative model. In order to characterize the limiting behavior of the proposed estimators, we make the following assumptions for all $i=1, \ldots, n$ and $m, l=1, \ldots, M$.

(A0) The dimension of the subject-specific parameters is $j$, where $j$ is known; $\theta_{i}^{*}$ is an interior point of $\Theta$, which is a compact subset of $\mathbb{R}^{j}$.

(A1) The state duration distributions $r_{m, i}($.$) has finite support.$

(A2) There exists $0<\sigma_{-} \leq \sigma_{+}<1$ such that $\sigma_{-} \leq P_{\theta_{i}^{*}}\left(S_{i}^{t+1}=l \mid S_{i}^{t}=m\right) \leq \sigma_{+}$, for $\mathrm{t}=1, \ldots, \mathrm{T}$; and $\sum_{\mathrm{m}=1}^{\mathrm{M}} P_{\theta_{i}^{*}}\left(Y_{i}^{t}=y \mid S_{i}^{t}=m\right)>0$ for all $\mathrm{y} \in \operatorname{suppY}_{\mathrm{i}}^{\mathrm{t}}$ and $\mathrm{t}=1, \ldots, \mathrm{T}$.

(A3) For each $\theta \in \Theta$ the transition kernel indexed by $\theta$ is Harris recurrent and aperiodic ${ }^{40-42}$.

(A4) The transition kernel is continuous in $\theta$ in an open neighborhood of $\theta_{i}^{*}$.

(A5) The hidden semi-Markov model is identifiable up to label-switching ${ }^{43,44}$.

These assumptions are standard in latent-state models ${ }^{45-49}$. Assumption (A0) avoids non-regularity occurring at boundary points; the assumption of a fixed dimension could be relaxed, for example, to the assumption that one has a strongly consistent estimator of $\mathrm{j}$. Assumption (A1) is common in semi-markov models and simplifies asymptotic arguments; if the finite support condition does not hold, one can use a nested sequence of finite approximations at the expense of more delicate asymptotic arguments ${ }^{50,51}$. Assumption (A4) is a regularity condition that avoids non-standard asymptotic behavior associated with non-smooth functionals ${ }^{52,53}$. Assumptions (A2), (A3), (A5) ensure that the model is well-defined; we show that (A5) holds for the zero-inflated Poisson semi-Markov model in the supplementary material. A proof of the following lemma and theorem is also provided in the supplementary material.

Lemma 1. Under (A0)-(A5), the MLE for the subject-specific hidden semi-Markov model in the first stage is strongly consistent, $\hat{\theta}_{i, T} \rightarrow \theta_{i}^{*}$ almost surely as $\mathrm{T} \rightarrow \infty$ for all $\mathrm{i}=1, \ldots, \mathrm{n}$.

Define $\Omega_{\mathrm{m}}^{*}=\left[\Omega_{\mathrm{m}, 0}^{*}, \Omega_{m, 1}^{*}\right]$ and the corresponding estimator $\hat{\Omega}_{\mathrm{m}, \mathrm{n}}=\left[\hat{\Omega}_{\mathrm{m}, 0, \mathrm{n}}, \hat{\Omega}_{m, 1, n}\right]$. Let $\Gamma_{\mathrm{m}}^{*}, \hat{\Gamma}_{m, n}$, $\Lambda_{m, l}, \hat{\Lambda}_{m, l, n}$ be defined analogously. Further, define the augmented design matrix $\tilde{W} \in \mathbb{R}^{n \times(d+1)}$ whose ith row is $\widetilde{W}_{i}=\left[1, W_{i}^{\prime}\right] \in \mathbb{R}^{d+1}$. Then the second stage regression models can be equivalently written as vec $\left(\hat{b}_{1, m, T}\right)=$ $(I \otimes \tilde{W}) \operatorname{vec}\left(\Omega_{m}\right)+v_{m}, \operatorname{vec}\left(\hat{c}_{1, m, T}\right)=(I \otimes \tilde{W}) \operatorname{vec}\left(\Gamma_{m}\right)+\eta_{m}, \operatorname{vec}\left(\hat{d}_{m, l, T}\right)=(I \otimes \tilde{W}) \operatorname{vec}\left(\Lambda_{m, l}\right)+\xi_{m, l}$, where vec is the vectorization operator, and $\nu_{\mathrm{m}}, \eta_{m}, \xi_{m, l}$ are independent mean zero residuals. Define $\tilde{B}=\mathrm{I} \otimes \tilde{W} \in \mathbb{R}^{\mathrm{qn} \times \mathrm{q}(\mathrm{d}+1)}$ whose ith row is $\tilde{B}_{i}$.

Theorem 3.2. Under (A0)-(A5), and further assume that $\mathrm{H}=\mathrm{n}^{-1} \lim _{\mathrm{n} \rightarrow} \tilde{W}^{\prime} W$ is a positive definite matrix. Then provided $\mathrm{T}, \mathrm{n} \rightarrow \infty$ with $\frac{\mathrm{n}}{\mathrm{T}} \rightarrow 0$, each of the following converges in distribution to a Gaussian distribution with mean zero and identity covariance:

$$
\begin{aligned}
& \left\{\left(\frac{1}{n} \tilde{B}^{\prime} \tilde{B}\right)^{-1}\left(\frac{1}{n} \tilde{B}^{\prime} \operatorname{diag}\left[\left\{\operatorname{vec}\left(\hat{b}_{1, m, T}\right)_{i}-\tilde{B}_{i}^{\prime} \operatorname{vec}\left(\hat{\Omega}_{m, \mathrm{n}}\right)\right\}^{2}\right]\left(\frac{1}{n} \tilde{B}^{\prime} \tilde{B}\right)^{-1}\right\}^{-\frac{1}{2}}\right. \\
& \quad \times \sqrt{\mathrm{n}}\left\{\operatorname{vec}\left(\hat{\Omega}_{\mathrm{m}, \mathrm{n}}\right)-\operatorname{vec}\left(\Omega_{\mathrm{m}}^{*}\right)\right\}, \\
& \quad\left\{\left(\frac{1}{n} \tilde{B}^{\prime} \tilde{B}\right)^{-1}\left(\frac{1}{n} \tilde{B}^{\prime} \operatorname{diag}\left[\left\{\operatorname{vec}\left(\hat{c}_{m, T}\right)_{i}-\tilde{B}_{i}^{\prime} \operatorname{vec}\left(\hat{\Gamma}_{m, \mathrm{n}}\right)\right\}^{2}\right]\left(\frac{1}{n} \tilde{B}^{\prime} \tilde{B}\right)^{-1}\right\}^{-\frac{1}{2}}\right. \\
& \quad \times \sqrt{\mathrm{n}}\left\{\operatorname{vec}\left(\hat{\Gamma}_{\mathrm{m}, \mathrm{n}}\right)-\operatorname{vec}\left(\Gamma_{\mathrm{m}}^{*}\right)\right\}, \\
& \left\{\left(\frac{1}{n} \tilde{B}^{\prime} \tilde{B}\right)^{-1}\left(\frac{1}{n} \tilde{B}^{\prime} \operatorname{diag}\left[\left\{\operatorname{vec}\left(d_{m, l, T}\right)_{i}-\tilde{B}_{i}^{\prime} \operatorname{vec}\left(\hat{\Lambda}_{m, \mathrm{l}, \mathrm{n}}\right)\right\}^{2}\right]\left(\frac{1}{n} \tilde{B}^{\prime} \tilde{B}\right)^{-1}\right\}^{-\frac{1}{2}}\right. \\
& \quad \times \sqrt{\mathrm{n}}\left\{\operatorname{vec}\left(\hat{\Lambda}_{\mathrm{m}, \mathrm{ln}, \mathrm{n}}\right)-\operatorname{vec}\left(\Lambda_{\mathrm{m}, \mathrm{l}}^{*}\right)\right\},
\end{aligned}
$$

for all $\mathrm{m}, \mathrm{l}=1, \ldots, \mathrm{M}$. 
In the preceding theorem, the requirement that $\frac{\mathrm{n}}{\mathrm{T}} \rightarrow 0$ is natural in applications using high-frequency accelerometer data where the number of observations per subject can be several orders of magnitude larger than the number of subjects. In the supplementary file 2, we have included extensive simulation experiments to evaluate the performance of the proposed treatment effect estimator.

Received: 10 November 2020; Accepted: 23 March 2021

Published online: 08 April 2021

\section{References}

1. National Research Council. Relieving pain in America: A blueprint for transforming prevention, care, education, and research. Washington, DC (2011).

2. Buckwalter, J. A., Saltzman, C. \& Brown, T. The impact of osteoarthritis: Implications for research. Clin. Orthop. Relat. Res. 427, 6-15 (2004).

3. Chen, A., Gupte, C., Akhtar, K., Smith, P. \& Cobb, J. The global economic cost of osteoarthritis: how the UK compares. Arthritis (2012).

4. Hadler, N. Osteoarthritis as a public health problem. Clin. Rheum Dis. 11, 175-185 (1985).

5. Elders, M. J. The increasing impact of arthritis on public health. J. Rheumatol. Suppl. 60, 6-8 (2000).

6. Hootman, J. M., Helmick, C. G. \& Bradley, J. T. A public health approach to addressing arthritis in older adults: The most common cause of disability. Am. J. Public Health 102, 426-433 (2012).

7. McClintock, M. K., Dale, W., Laumann, E. O. \& Waite, L. Empirical redefinition of comprehensive health and well-being in the older adults of the United States. Proc. Nat. Acad. Sci. 113, 3071-3080 (2016).

8. Park, J., Mendy, A. \& Vieira, R. Various types of arthritis in the united states: Prevalence and age-related trends from 1999 to 2014. Am. J. Public Health 108, 256-258 (2018).

9. Vierck, C. J., Hannson, P. T. \& Yezierski, R. P. Clinical and pre-clinical pain assessment: Are we measuring the same thing?. Pain 135, 7-10 (2008).

10. Mogil, J. S. Animal models of pain: Progress and challenges. Nat. Rev. Neurosci. 10, 283-294 (2009).

11. Hayes, A. G., Arendt-Nielsen, L. \& Tate, S. Multiple mechanism have been tested in pain: How can we improve the chances of success?. Curr. Opin. Pharmacol. 14, 11-17 (2014).

12. Lascelles, B. D. X., Brown, D. C., Maixner, W. \& Mogil, J. S. Spontaneous painful disease in companion animals can facilitate the development of chronic pain therapies for humans. Osteoarthr. Cartil. 26, 175-183 (2017).

13. Lascelles, B. D. X. et al. Cross-sectional study of the prevalence of radiographic degenerative joint disease in domesticated cats. Vet. Surg. 39, 535-544 (2010).

14. Slingerland, L., Hazewinkel, H., Meij, B. P., Picavet, P. \& Voorhout, G. Cross-sectional study of the prevalanece of clinical feature of osteoarthritis in 100 cats. Vet. J. 187, 304-309 (2011).

15. Gruen, M. E., Griffith, E. H., Thomson, A. E., Simpson, W. \& Lascelles, B. D. X. Criterion validation testing of clinical metrology instruments for measuring degenerative joint disease associated mobility impairment in cats. PLoS ONE 10(7), e0131839 (2015).

16. Lascelles, B. D. X. et al. Evaluation of a digitally integrated accelerometer-based activity monitor for the measurement of activity in cats. Vet. Anaesth. Analg. 35, 173-183 (2008).

17. Gruen, M. E. et al. A feline-specific anti-nerve growth factor antibody improves mobility in cats with degenerative joint diseaseassociated pain: A pilot proof of concept study. J. Vet. Int. Med. 30, 1138-1148 (2016).

18. Gruen, M. E. et al. The use of functional data analysis to evaluate activity in a spontaneous model of degenerative joint disease associated pain in cats. PLoS ONE 12(1), e0169576 (2017).

19. He, J., Li, H. \& Tan, J. Real-time daily activity classification with wireless sensor networks using hidden markov model. in Engineering in Medicine and Biology Society, 29th Annual International Conference of the IEEE, 3192-3195 (2007).

20. Mannini, A. \& Sabatini, A. M. Machine learning method for classifying human physical activity from on-body accelerometers. Sensors 10, 1154-1175 (2010).

21. Nickel C., Busch, C., Rangarajan, S. \& Mobius, M. Using hidden markov models for accelerometer-based biometric gait recognition. in Signal Processing and its Applications, IEEE 7th International Colloquium, 58-63 (2011).

22. Lee, Y. \& Cho, S. Activity recognition using hierarchical hidden markov models on a smartphone with $3 \mathrm{~d}$ accelerometer. Hybrid Artif. Intell. Syst. 6678, 460-467 (2011).

23. Ronao, C. A. \& Cho, S. Human activity recognition using smartphone sensors with two-stage continuous hidden markov models. in International Conference on Natural Computation, 10th International Conference of the IEEE, 681-686 (2014).

24. Scott, S. L., James, G. M. \& Sugar, C. A. Hidden markov models for longitudinal comparisons. J. Am. Stat. Assoc. 100, 359-369 (2005).

25. Shirley, K. E., Small, D. S., Lynch, K. G., Matiso, S. A. \& Oslin, D. W. Hidden markov models for alcoholism treatment trial data. Ann. Appl. Stat. 4, 366-395 (2010).

26. DeSantis, S. M. \& Bandyopadhyay, D. Hidden markov models for zero-inflated poisson counts with an application to substance use. Stat. Med. 30, 1678-1694 (2011).

27. Olteanu, M. \& Ridgway, J. Hidden markov models for time series of counts with excess zeros. in European Symposium on Artificial Neural Networks, 2012 Proceedings, 133-138 (2012).

28. Ferguson, J. D. Variable duration models for speech. in Proceedings of the Symposium on the Application of HMMs to Text and Speech, 143-179 (1980).

29. Russel, M. \& Moore, R. Explicit modelling of state occupancy in hidden Markov models for automatic speech recognition. in Acoustics, Speech, and Signal Processing, IEEE International Conference on ICCASSP. Volume 10, 5-8 (1985).

30. Levinson, S. E. Continuously variable duration hidden markov models for automatic speech recognition. Comput. Speech Lang. 1, 29-45 (1986).

31. Stukel, T. A. \& Demidenko, E. Two-stage method of estimation for general linear growth curve models. Biometrics 53, 720-728 (1997).

32. Hanushek, E. A. Efficient estimators for regressing regression coefficients. Am. Stat. 28, 66-67 (1974).

33. Gruen, M. E., Thomson, A., Simpson, W. \& Lascelles, B. D. X. Detection of clinically relevant pain relief in cats with degenerative joint disease associated pain. J. Vet. Int. Med. 28, 346-350 (2014).

34. Viterbi, A. Error bounds for convolutional codes and an asymptotically optimum decoding algorithm. IEEE Trans. Inf. Theory 13, 260-269 (1967).

35. van Kuppevelt, D. et al. Segmenting accelerometer data from daily life with unsupervised machine learning. PLoS ONE 14(1), e0208692 (2019).

36. Huang, Q. et al. Hidden markov models for monitoring circadian rhythmicity in telemetric activity data. J. R. Soc. Interface 15, 20170885 (2018).

37. Diggle, P. Analysis of Longitudinal Data (Oxford University Press, Oxford, 2002). 
38. Verbeke, G. \& Molenberghs, G. Linear Mixed Models for Longitudinal Data (Springer, Berlin, 2009).

39. Rabiner, L. R. A tutorial on hidden Markov models and selected applications in speech recognition. Proc. IEEE 77, 257-286 (1989).

40. Schwarz, G. et al. Estimating the dimension of a model. Ann Stat 6, 461-464 (1978).

41. Athreya, K. B. \& Lahiri, S. N. Measure Theory and Probability Theory (Springer, 2006).

42. Meyn, S. P. \& Tweedie, R. L. Markov chains and stochastic stability (Springer, 2012).

43. Allman, E. S. et al. Identifiability of parameters in latent structure models with many observed variables. Ann. Stat. 37(6A), 3099-3132 (2009).

44. Gassiat, E., Cleynen, A. \& Robin, S. Finite state space non parametric hidden Markov models are in general identifiable. arXiv: 1306.4657 (2013).

45. Leroux, B. G. Maximum-likelihood estimation for hidden Markov models. Stoch. Process Their Appl. 40, 127-143 (1992).

46. Bickel, P. J. et al. Asymptotic normality of the maximum likelihood estimator for general hidden Markov models. Ann. Stat. 26, 1614-1635 (1998).

47. Le Gland, F. \& Mevel, L. Exponential forgetting and geometric ergodicity in hidden markov models. Math. Control Signals Syst. 13, 63-93 (2000).

48. Douc, R. et al. Asymptotics of the maximum likelihood estimator for general hidden Markov models. Bernoulli 7, 381-420 (2001).

49. Douc, R. et al. Asymptotic properties of the maximum likelihood estimator in autoregressive models with Markov regime. Ann. Stat. 32, 2254-2304 (2004).

50. Barbu, V. S. \& Limnios, N. Semi-Markov chains and hidden semi-Markov models toward applications: their use in reliability and DNA analysis (Springer, 2009).

51. Trevezas, S. \& Limnios, N. Exact mle and asymptotic properties for nonparametric semi-Markov models. J. Nonparametr. Stat. 23, 719-739 (2011).

52. Van der Vaart, A. On differentiable functionals. Ann. Stat. 19, 178-204 (1991).

53. Potscher, B. M. \& Prucha, I. R. Dynamic nonlinear econometric models: Asymptotic theory (Springer, 2013).

\section{Acknowledgements}

Laber acknowledges support from the National Institutes of Health (P01 CA142538) and the National Science Foundation (DMS-1555141, DMS-1513579). Staicu acknowledges support from the National Science Foundation (DMS-1454942). Funding for the original study was provided by a grant (45079) from Boehringer-Ingelheim Vetmedica, Inc.

\section{Author contributions}

B.D.X.L. designed the original study; B.D.X.L. performed the original study; A.-M.L., E.L., Z.X. and B.D.X.L. designed the current study. Z.X., A.M.-L. and E.L. performed the analyses and prepared the figures; Z.X. and B.D.X.L. drafted the manuscript; all authors reviewed the manuscript.

\section{Competing interests}

BDXL has participated in sponsored CE for Boehringer-Ingelheim (manufacturers of Metacam used in the original study). A-MS, EL and ZX have no conflicts of interest to declare.

\section{Additional information}

Supplementary Information The online version contains supplementary material available at https:/doi.org/ 10.1038/s41598-021-87304-w.

Correspondence and requests for materials should be addressed to B.D.X.L.

Reprints and permissions information is available at www.nature.com/reprints.

Publisher's note Springer Nature remains neutral with regard to jurisdictional claims in published maps and institutional affiliations.

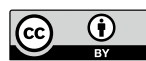

Open Access This article is licensed under a Creative Commons Attribution 4.0 International License, which permits use, sharing, adaptation, distribution and reproduction in any medium or format, as long as you give appropriate credit to the original author(s) and the source, provide a link to the Creative Commons licence, and indicate if changes were made. The images or other third party material in this article are included in the article's Creative Commons licence, unless indicated otherwise in a credit line to the material. If material is not included in the article's Creative Commons licence and your intended use is not permitted by statutory regulation or exceeds the permitted use, you will need to obtain permission directly from the copyright holder. To view a copy of this licence, visit http://creativecommons.org/licenses/by/4.0/.

(C) The Author(s) 2021 